\title{
émulations
}

\section{Incertaine est la nuit dakaroise}

\section{Quand les possibles nocturnes défient le réel diurne}

\author{
Thomas Fouquet
}

Émulations - Revue de sciences sociales

2020, n 33, "La nuit urbaine. Un espace-temps complexe entre opportunités et inégalités ».

\section{Article disponible à l'adresse suivante}

https://ojs.uclouvain.be/index.php/emulations/article/view/fouquet

\section{Pour citer cet article}

Thomas Fouquet, «Incertaine est la nuit dakaroise. Quand les possibles nocturnes défient le réel diurne », Émulations, n 33, Mise en ligne le 15 juin 2020.

DOI : 10.14428/emulations.033.02

Distribution électronique : Université catholique de Louvain (Belgique) : ojs.uclouvain.be

(C) Cet article est mis à disposition selon les termes de la Licence Creative Commons Attribution, Pas d'Utilisation Commerciale 4.0 International. http://creativecommons.org/licenses/by-nc/4.0/

Éditeur : Émulations - Revue de sciences sociales / Presses universitaires de Louvain https://ojs.uclouvain.be/index.php/emulations

ISSN électronique : 1784-5734

PUL PRESSES UNIVERSITAIRES DE LOUVAIN 


\title{
Incertaine est la nuit dakaroise
}

\section{Quand les possibles nocturnes défient le réel diurne}

Thomas Fouquet $^{1}$

\begin{abstract}
[Résumé] En prenant Dakar pour site ethnographique, cette étude envisage la nuit urbaine sous l'angle du régime de l'incertain et de l'indéterminé qu'elle emporte avec elle. Cette dimension d'incertitude est d'abord remise en perspective de dynamiques de « liquéfaction » qui caractérisent plus généralement nos temps présents. Les interprétations proposées démontrent que ce que l'on nomme génériquement l'incertain renvoie à des enjeux hétérogènes et appelle un examen plus fin, à la lumière de configurations sociohistoriques propres à chaque contexte urbain d'abord, en regard des durées ou des temporalités qui s'y rapportent ensuite, du point de vue des usages sociaux incidents enfin. Ces réflexions sont ensuite approfondies à travers l'articulation des notions de nocturnité et de mobilité pour ce qui touche en particulier à l'idée d'un changement en train de se faire, et dont l'issue est par définition incertaine et indéterminée.
\end{abstract}

Mots-clés : nuit, urbain, Dakar, incertitude, mobilités.

Uncertain is the Dakar-by-night.

When the nocturnal possibilities challenge the diurnal reality

[Abstract] Takin Dakar as an ethnographic site, this article considers the topic of urban night in the light of uncertainty and indetermination, notably by relocating this questioning in the broader perspective of "liquefaction" dynamics that characterize our present times. In the urban Senegalese context, what is generically termed "uncertain" appears intrinsically heterogeneous and calls for a more detailed examination, in connection with local sociohistorical configurations, and with regard to the concrete experiences and social uses of (nocturnal) uncertainty. These reflections are then deepened through a joint approach of the two notions of nocturnality and mobility, in particular with regard to the idea of a change in progress and thus, by definition, uncertain and indeterminate.

Keywords: night, urban, Dakar, uncertainty, mobilities.

\section{Introduction}

À Dakar, l’univers des loisirs nocturnes est fréquemment associé à une idée de (dé)perdition sociale et culturelle, voire de dévoiement moral. En témoignent pléthore d'articles de presse volontiers sensationnalistes, mais aussi les offensives fréquemment portées par des " entrepreneurs de morale », en particulier dans le registre religieux, contre un certain nombre de pratiques que le Dakar-by-night est réputé héberger, voire favoriser : consommation d'alcool, intimités sexuelles éventuellement tarifées, accoutrements et danses considérés comme " peu vertueux ", voire tout à fait scandaleux, etc. Cette stigmatisation du nocturne traduit une certaine « angoisse morale ${ }^{2}$ », somme

\footnotetext{
${ }^{1}$ Laboratoire IMAf/LMI MOVIDA CNRS, Aubervilliers, France.

${ }^{2}$ Cette notion définit ici le sentiment d'inquiétude et/ou de réprobation qu'inspire un groupe spécifique - ou en tout cas socialement construit comme tel -, un type d'activités, voire de lieux ou même de temps (nocturne en l'espèce) où elles se déroulent, pour ce qu'ils semblent constituer
} 
toute classique du traitement social et politique dont la nuit fait l'objet à Dakar comme ailleurs (voir notamment Cabantous, 2009; Chatterton, 2002 ; Paquot, 2000).

Dans le contexte sénégalais, ces dénonciations peuvent au demeurant étayer une critique bien plus ample, adressée à des modèles sociaux et culturels venus d'ailleurs et censés influencer négativement la jeunesse sénégalaise. Qu’il s'agisse de manières de se vêtir et de parler, d'usages du corps et de la sexualité ou, en termes plus généraux, de façons de se comporter et de se produire publiquement, les nuits urbaines sont vues comme de haut-lieux d'appropriation de styles, de modèles et de pratiques importés, et de ce fait frappés de jugements d'illégitimité, voire de dangerosité morale. Ce stigmate nocturne s'applique avec davantage de force aux femmes, comme l'indique bien l'expression commune de « fille qui sort la nuit » (du wolof : «jiggéen yu gën guddi ») qui véhicule un présupposé de débauche, voire fonctionne comme une (d)énonciation parabolique de la prostitution : rechercher la « bonne vie » dans le monde nocturne peut exposer frontalement à des jugements de femme de « mauvaise vie».

Plus généralement, les postures sociales adoptées la nuit durant sont fréquemment assimilées à des impostures culturelles. Suivant la vulgate « de la perte » qui se dessine ici, pactiser culturellement avec les mondes occidentaux exposerait au risque de se couper d'une véracité sociale et culturelle, avec toutes les conséquences supposément néfastes et perverses que cela comprend. Achille Mbembe a dévoilé certains fondements des logiques évoquées ici : «Dans le discours canonique africain, faire mémoire de la colonie, c'est presque toujours se souvenir d'un décentrement primordial entre le moi et le sujet. » Enrayer ce processus au fil duquel «le moi authentique serait devenu un autre " impliquerait que "soit restaurée chez le sujet une matrice symbolique originelle (la tradition)» (Mbembe, 2006 : 104). Sous cette optique, le rapport à l'idée de modernité prend les formes d'un terrain de contestation où s'affrontent des visions du monde et de la place qu'on y occupe. De fait, aux yeux de nombreux citadins, l'attrait de la vie nocturne tient justement pour une bonne mesure au truchement qu'elle offre vers quelque chose de plus vaste qu'un hic et nunc largement vécu du côté du manque, de la privation, ou encore du blocage social comme géographique. La fréquentation d'établissements de nuit évocateurs d'un cosmopolitisme urbain (Fouquet, 2018) est alors susceptible d'offrir un prolongement local, mais aussi tangible, à cette soif du plus vaste assimilable à une "globalisation des rêves »(Geschiere, Rowlands, 1996). Celle-ci prend du reste plutôt la forme d'une « onirisation du global » dans un contexte où l'ampleur des aspirations migratoires n'a d'égal que les difficultés, voire l'impossibilité à les réaliser.

Dans les deux cas de figure ébauchés ici - la supposée dangerosité de la ville nocturne versus les opportunités d'ouverture qu'elle offrirait -, la nuit urbaine est conçue comme un site emblématique de circulation et de domestication de tendances mondialisées, suivant néanmoins des visions et jugements tout à fait opposés. Ces perceptions très polarisées se rapprochent de celles qui ont longtemps prévalu au sujet des

une menace contre les valeurs et les intérêts dominants. Cette notion fait en ce sens directement écho à celle de moral panic (voir notamment Cohen, 2002 [1972]). 
villes plus généralement, tantôt teintées de peurs et de négativité (notamment Paquot, 2000), tantôt louées pour les opportunités qu'elles offriraient alors que « l'air de la ville rend libre » suivant cet ancien adage re-popularisé par Max Weber (2014 : 74). En contextes africains, de tels jugements font sens également en regard de l'opposition entre, d'un côté, mondes villageois (ré)enchantés et, de l'autre, centres urbains assimilés à des styles de vie délétères, car importés ou imposés au fil de l'expérience coloniale (Diouf, 2013 ; Fourchard, 2004). Ces configurations apparaissent néanmoins exacerbées au chapitre de la nuit urbaine et on peut alors suggérer que les jugements qui s'y adressent réactivent et actualisent le vieux binarisme tradition versus modernité, au fil de débats contradictoires de la modernité dont l'univers nocturne constitue une arène privilégiée.

Une tension se dessine ici, entre le désirable (la nuit est riche de promesses) et le détestable (elle est fondamentalement menaçante) ; mais aussi entre l'ordinaire (la nuit revient chaque jour) et l'extraordinaire (elle reste néanmoins singulière compte tenu non seulement des usages hors-norme dont elle ferait l'objet, mais aussi et surtout des imaginaires qui s'y greffent). Ces configurations ambivalentes jouent un rôle central dans la fabrication de la nuit urbaine comme un univers social, symbolique et éventuellement politique distinct. Par ailleurs, on peut observer que ces appréciations divergentes, voire antagoniques, s'organisent in fine en narrations concurrentes de la bonne urbanité ; nocturnité ${ }^{3}$ et urbanités s'en trouvent en retour étroitement liées, la première servant en quelque sorte de révélateur aux secondes en les situant dans une épaisseur sociale, culturelle et politique plus importante.

Je propose ici d'approfondir cette dimension d'ambivalence de la nuit urbaine, en prenant Dakar pour site ethnographique principal et en centrant mes réflexions sur la question de l'incertitude du nocturne. Cet angle d'approche m'apparaît fécond à au moins deux titres : l'incertain singularise la nuit en la définissant comme un espace-temps de désorganisation des repères établis ; dans le même mouvement, il la départicularise en la plaçant au cœur d'une discussion plus ample sur la nature des temps présents, c'est-à-dire le sentiment (collectif) d’indétermination qui caractériserait la période actuelle.

Depuis une dizaine d'années, tout un courant de recherches s'intéresse aux manières par lesquelles les sociétés contemporaines sont ou paraissent soumises à l'inconstant et à l'inconsistant, sous l'effet de l'affaiblissement de structures de sens élaborées dans la durée et du développement incident d'une trame de l'incertain (voir notamment Bauman, 2007 ; Bauman, 2013 ; Cooper, Pratten, 2015 ; Kleist, Thorsen, 2017 ; PenaVega, 2014 ; Sardar, 2010). La remise en question des métarécits du progrès et de la croissance économique, le recul voire l'obsolescence des idéologies politiques et, plus fondamentalement, la volatilité accrue des repères sur lesquels de nombreux individus s'appuient dans la conduite de leur existence indiqueraient ainsi l'avènement de

\footnotetext{
${ }^{3}$ En termes généraux, cette notion peut être définie comme l'ensemble des représentations et des
} pratiques, y compris discursives, qui organisent le rapport à la nuit dans un monde social donné. 
« temps postnormaux »(Sardar, 2010). Non pas qu'une normalité antérieure pourrait être définie a contrario de façon absolument homogène et stable, de surcroît égale à elle-même partout et aux yeux de tous ${ }^{4}$. Plutôt, un nombre toujours croissant de femmes et d'hommes ne peuvent que réfuter ces grands récits tant leurs réalités quotidiennes en démentent les allégations matérielles et économiques, comme idéologiques et politiques. En ce sens, « l'incertitude est devenue un trope dominant, une force inévitable » qui accompagne et entoure les individus dans leurs expériences subjectives quotidiennes (Cooper, Pratten, 2015:1).

S'il n'est pas question ici d'entrer dans le détail de ces discussions, on peut admettre que la nuit se caractérise par un analogue bousculement des repères, c'est-à-dire qu'elle porte la faculté de remodeler dans une sorte d'indétermination certaines évidences que le diurne charrie. Ces processus peuvent s'adosser à des facteurs relativement palpables - au niveau des "sensorialités nocturnes » et des ambiances qui les sous-tendent (Labussière, 2013 ; Nativel, 2016), en termes de reconfiguration du contrôle social et politique, etc. - tout autant qu'ils intègrent les liens avant tout psychoaffectifs que les individus entretiennent à leur environnement - avoir le sentiment que la nuit est porteuse d'heureuses promesses ou au contraire de redoutables menaces. Mais dans tous les cas, la nuit participerait d'un renforcement de l'incertain, sur des séquences temporelles bien sûr beaucoup plus brèves, c'est-à-dire en un passage chaque jour réitéré d'un monde (diurne/nocturne) à l'autre. Au demeurant, l'idée d'une reconfiguration de l'intelligible, du prévisible ou encore du saisissable est bien là, en quelque sorte échantillonnée et encapsulée dans les temporalités quotidiennes. En hypothèse, cette incertitude propre à la nuit est comme potentialisée en milieux urbains alors que la grande ville est par essence « un site d'imprévisibilité endémique » (Bauman, 2013:123) en tant qu'elle se caractérise par la coprésence d'une foule d'individus pour la plupart étrangers les un·e·s aux autres, avec ce que cela peut comporter de menaces réelles ou fantasmées, mais aussi d’opportunités - effectives ou espérées.

\section{Sur l'incertitude en général, et celles dakaroises en particulier : penser avec (et au-delà de) l'incertain}

La nuit urbaine s'impose ainsi comme un laboratoire privilégié pour l'édification d'« un nouveau paradigme incorporant l'incertitude au cœur de ses principes » (Pena-Vega, 2014: 5). Pour être recevable, cette perspective de recherche programmatique doit intégrer l'épaisseur sociohistorique des situations locales, ce que l'on pourrait nommer ici la trame singulière de l'incertain de Dakar.

On peut d'emblée souligner que du point de vue de nombreux habitants de Dakar, ce qui est normal c'est que rien ne l'est vraiment au fond, tant la capacité à prédire son devenir y compris le plus immédiat, et surtout à avoir prise sur lui, s'avère problématique. L'incertitude constitue en ce sens un important vecteur de subjectivation :

\footnotetext{
${ }^{4}$ Sur la question plus large - et néanmoins connexe - des compréhensions et narrations « autres »
} de la modernité, voir notamment Gaonkar (2001). 
les individus en sont faits, tout autant qu'ils font avec. À une échelle sociopolitique et historique plus macro, les entreprises postindépendances de "construction nationale » (Cruise O’Brien, Diop, Diouf, 2002), puis la téléologie du développement tout autant que celle de l'« Émergence africaine ${ }^{5}$ » qui en représente la rhétorique la plus actuelle, ne font pas ou plus le poids face aux conditions de vie effectives d'une majorité de ceux censés bénéficier de ces grands programmes. Les réalités quotidiennes du plus grand nombre d'individus se déclinent localement sur le modèle de l'entrave et du manque. Au niveau international, c'est la figure de l'endiguement qui s'impose, compte tenu des barrières quasi insurmontables qui sont opposées aux candidats à l'émigration, celle-ci étant pourtant devenue le principal sinon l'unique levier de mobilité sociale aux yeux de beaucoup.

À Dakar, si l'on excepte une petite minorité de privilégié·e.s en mesure de véritablement contrôler leur présent, voire leur devenir ${ }^{6}$, on observe ainsi que le faisceau des empêchements ou des complications de tous ordres - familiaux, professionnels, administratifs, etc. - est si large que les facultés d'anticipation ou de projection individuelles s'en trouvent largement contraintes. Ceci emporte assurément des effets quant à la « capacité à aspirer » (Appadurai, 2004), c'est-à-dire notamment quant à l'édification intime d'un rapport au devenir individuel, qui peut être tantôt atrophié - un no future - et tantôt hypertrophié - la défection au moins psychoaffective vis-à-vis d'un présent privé de sens ou d'horizon, au profit du surinvestissement d'un à-venir fantasmé.

Cette normalité-là est donc vécue avant tout au rythme de l'aléatoire et des aléas, pouvant confiner à un « présentisme » (Hartog, 2003) écrasant. Mes recherches consacrées aux aspirations migratoires de jeunes Dakarois (notamment Fouquet, 2008) démontrent que l'incertitude liée à un avenir pour le moins évasif et le sentiment de blocage - ou waithood suivant la formule d'Alcinda Honwana (2012) - y occupent une place également prépondérante. Ainsi, ce jeune habitant de la banlieue de Dakar dressait le constat suivant :

- Tu te lèves le matin et tu ne sais jamais ce que la journée te réserve : tu vas trouver un peu d'argent, ou pas? On va te coller telle ou telle corvée familiale, ou pas? Tu vas être bloqué dans les embouteillages pendant des heures, ou pas? Tu vas apprendre le décès d'un proche, ou pas ? Et puis toi-même, tu vas mourir, ou pas? Je vais arrêter là, mais c'est juste pour dire que tu ne sais jamais ce qui t'attend en fait.

\footnotetext{
${ }^{5}$ La notion d'« Émergence » s'est imposée aujourd'hui comme le maître-mot de différents gouvernants, notamment en Afrique, pour qualifier les trajectoires à venir ou tout au moins attendues de leurs États. Cette rhétorique de l'Émergence vient se suppléer à celle du développement en revêtant, à certains égards, une dimension de marketing politique, à l'instar dudit « Plan Sénégal émergent » (PSE). En ligne, consulté le 14 novembre 2019. URL: https://www.sentresor.org/app/ uploads/pap2_pse.pdf. Pour une genèse critique de la notion d'Émergence, voir Chaouad (2016). 6 J'évoque ici, en termes généraux, la possibilité de ne pas avoir à dépendre des autres pour la gestion (notamment économique) du quotidien, dans un contexte où la protection sociale étatique n'existe pas, et où le groupe au sens large reste donc le meilleur voire le seul rempart face aux aléas de l'existence.
} 
- Thomas Fouquet (TF) : D'accord, mais tout ce dont tu parles, on le retrouve partout, pas seulement au Sénégal. Personne ne peut vraiment deviner par avance ce qui va lui arriver.

- Oui, je ne te dis pas le contraire. Mais chez vous [en France, en Europe], si tu es honnête, tu dois reconnaître que vous pouvez quand même prévoir un peu. Par exemple, si tu as un rendez-vous, tu vas tout faire pour arriver à l'heure, ou bien même pour y aller, n'est-ce pas ? Je suis d'accord qu'il peut y avoir des petites choses imprévues, mais dans l'ensemble ce que tu prévois de faire, c'est solide, non ?! Ici, au Sénégal, c'est tout le contraire en fait, il n'y a jamais rien de clair ou de facile. Tu vois, c'est ça qui est compliqué, c'est très lourd même. On ne peut jamais s'organiser un petit peu, parce qu'il y a toujours des choses qui vont être contre toi. Si je me lève le matin et que je me dis : « aujourd'hui j'ai ça, ça et ça à faire pour avancer un peu », tu peux être sûr que ça ne va jamais se passer comme j'avais prévu. C'est le bordel partout, tu ne peux pas avoir confiance dans quoi que ce soit. Et surtout, surtout!, tu ne vas jamais faire tes trucs comme tu aurais envie de les faire, parce qu'il y aura toujours quelque chose qui va te freiner ou t'emmener ailleurs. [...] J'ai beaucoup d'amis qui sont découragés, ils ne font plus rien en fait, ils restent le cul posé toute la journée parce que, tu vois, ça te fatigue trop, tout ça. Ils attendent que ça leur tombe du ciel parce qu'ils pensent qu'ils ne pourront rien obtenir autrement... Ou bien ils attendent juste de partir, de quitter le Sénégal quoi, de n'importe quelle manière. Si les gens sont prêts à se noyer dans la mer, c'est pour le plaisir, tu crois ? C'est juste qu'ils n'ont pas le choix, hein. Donc tu vois, prier ou partir [en migration] c'est pareil en fait : c'est des croyances, rien de solide en tout cas, que des espérances. Espérer, en fait, je me dis qu'il n'y a que ça qui nous reste. [...] On nous dit que nous, les jeunes, nous sommes des fainéants, qu'on ne veut rien foutre, mais c'est des conneries ça, c'est juste du découragement en fait! C'est ça qui nous bloque, ici, on n'est jamais libres de faire ce qu'on veut ${ }^{7}$.

À la lumière de ce type de discours et des expériences qui les sous-tendent, le territoire citadin s'assimile peut-être avant tout à un « territoire de l'attente »(Vidal, Musset, 2015). La ville constitue le cadre englobant où ces situations de blocage se formalisent spatialement tout autant que socialement et culturellement, non pas nécessairement sous le signe de la stricte vacuité ou sous l'effet d'un attentisme mortifère, mais plutôt à travers la tension entre désirs de mobilité (sociale et géographique) et immobilisme contraint.

C’est en prise avec de tels constats que, depuis la fin des années 1990, je conduis mes recherches à Dakar sur les désirs d'Ailleurs de jeunes citadins. Entre autres questions guidant mes travaux : comment construit-on sa place ici lorsque l'on est tout entier pris dans le désir d'un ailleurs, social et/ou géographique ? Et en prolongement, quels usages et conceptions du présent émergent face à la perspective d'un avenir que l'on sait ou suppose par avance profondément insatisfaisant?

${ }^{7}$ Homme, 25 ans, Dakar, 2014. Entretien conduit en wolof et français. Dans la suite du texte, les extraits d'entretien cités seront pareillement renseignés du point de vue du sexe de l'interlocuteur, de son âge, du lieu de l'entretien et de l'année, de/des langue·s d'interlocution enfin. 
Dans mes travaux, ces configurations sont rendues particulièrement visibles parmi de jeunes citadines, pour la plupart originaires de Dakar et de sa banlieue, qui fréquentent quotidiennement des bars et night-clubs de la capitale sénégalaise en quête de fortunes diverses, notamment migratoires ${ }^{8}$. J'ai conduit une ethnographie étendue sur une période cumulée de plus de cinq ans auprès de ces jeunes femmes, en mêlant une approche classique de type observation participante - qu'il m'a fallu néanmoins adapter au contexte urbain nocturne ${ }^{9}$ - et entretiens ethnographiques, auxquels j'ai associé un travail de recueil biographique approfondi auprès d'une dizaine d'interlocutrices, afin notamment de retracer des " carrières de la nuit ${ }^{10}$ ». Cette approche anthropologique a été renforcée par un travail historique visant à appréhender, notamment, les modes de constitution des jugements sociaux qui s'adressent aux jeunes femmes « libres » en milieux urbains ouest-africains depuis les années 1940 (Fouquet, 2011).

À Dakar, j’ai nommé mes interlocutrices des " aventurières de la nuit » par référence aux concepts d'aventure et d'aventurier qui se sont imposés dans le champ des études africaines et migratoires au cours des vingt dernières années (Bredeloup, 2014). L’opportunité d'un départ en migration occupe effectivement une place centrale dans ces pratiques, en se concrétisant à travers notamment la fréquentation d'établissements de nuit très internationalisés et la possibilité incidente de rencontrer un partenaire étranger, accointance - « amoureuse »/intime ou autre - susceptible de faciliter les démarches migratoires. Mais comme je m’y réfère, l'approche par l'aventure renvoie également aux usages hors-norme et indociles de la ville nocturne et aux désirs de mobilité sociale et géographique qui les sous-tendent. À travers des pratiques qui mêlent étroitement transactions économico-sexuelles, négociation de la valeur individuelle et recherche de pouvoir personnel, les jeunes femmes " aventurières de la nuit » investissent le Dakar-by-night comme une scène expressive (Irwin, 1977) où d'autres destins sont susceptibles de trouver un mode de concrétisation ou au moins de figuration, ne serait-ce qu'au travers de « mobilités ascendantes fictives » (Mitchell, 1996 [1956] : 225).

\footnotetext{
${ }^{8}$ Ce travail a d'abord fait l'objet d'un doctorat en anthropologie (Fouquet, 2011) ; je l'ai depuis approfondi, notamment en mettant plus fermement l'accent sur le contexte urbain nocturne englobant.

${ }_{9}^{9}$ Si la scène urbaine nocturne (festive en l'espèce) se prête remarquablement au travail d'observation, elle rend en revanche bien plus complexes les échanges verbaux formalisés, pour différentes raisons : disponibilité des acteurs, niveau sonore, types d'interaction, etc. L'objet nocturne rabat alors dans une large mesure la séparation des méthodes entre l'alternance jour (entretiens)/nuit (observations). Cette distinction entre le nocturne et le diurne, sur les plans méthodologique et épistémologique, s'est avérée très féconde in fine: la mise en regard et en tension des pratiques nocturnes que j'observais quotidiennement, avec les discours que les acteurs en produisent $a$ priori ou a posteriori, est bien révélatrice de modes subjectifs d'être à la nuit. L'écart éventuel entre le vécu et l'observé, d'une part, et le narré d'autre part, devient en ce sens un levier interprétatif qui se déploie lui aussi entre jours et nuits; il ouvre simultanément une réflexion très riche sur les liens entre narrations verbales et non verbales.

10 Le problème de la «participation » dans le milieu nocturne enquêté a soulevé avec acuité et persistance celui de ma place, en tant qu'ethnographe homme, Occidental, auprès de femmes, Africaines. J'ai eu à aborder cette question de l'épistémologie de l'enquête notamment dans Fouquet (2014a).
} 
Quoi qu'il en soit de l'effectivité de ces déplacements positionnels, qui ne sont de toute façon pas sans conséquence sur les plans sociaux, économiques, mais aussi bien sûr intimes, il reste que la nuit est vécue comme un temps ouvert, ou qui ouvre. Cette idée peut être comprise de deux façons complémentaires. D’abord, cette ouverture permet de conjurer temporairement le sentiment de blocage, voire d'insignifiance sociale, donc dans un rapport symétriquement opposé à la finitude et aux empêchements rattachés au diurne, avec tout le potentiel critique que cela recouvre. On peut ensuite évoquer le désir d'accéder à des « mondes plus vastes » (Simone, 2005-2006) qui imprègne les usages de la nuit parmi ces jeunes femmes. Ceci se traduit notamment dans la fréquentation d'établissements de nuit qui se caractérisent par le brassage d'individus issus d'horizons socioéconomiques, culturels et géographiques divers, par contraste avec les lieux considérés comme trop locaux ou «trop sénégalais ». De ce point de vue, la géographie nocturne de Dakar paraît traversée de lignes de démarcation internes assimilables à celles distinguant les «Locaux » des « Cosmopolites » (Hannerz, 1990). La formule «trop sénégalais » condamne sans appel un établissement considéré comme bas de gamme, trop ancien et/ou dénué d'élégance et de chic, à l'instar des maquis ivoiriens ou des nganda congolais, par exemple. On jugera ainsi négativement les établissements qui ne jouent que de la musique africaine ou de la salsa pour une clientèle d'âge mûr dans un décor vétuste peu conforme aux goûts du jour ${ }^{11}$, au profit de sonorités internationales - hip-hop et black music singulièrement (Fouquet, 2014b) - et de décorations d'intérieur plus rutilantes ou avant-gardistes.

Ces configurations se traduisent également à une plus grande échelle, sous l'angle de la constitution des centralités nocturnes de Dakar. En particulier, la zone dite de NgorAlmadies, située à l'extrême ouest de la presqu'île du Cap-Vert délimitant la région de Dakar, faiblement urbanisée jusqu'au début des années 1990, rassemble aujourd'hui des institutions et firmes internationales, une population composée pour une bonne part d'expatriés occupant des résidences de haut standing, mais aussi et surtout une économie du loisir nocturne dense et internationalisée. Elle matérialise en ce sens une certaine idée de la modernité urbaine, connectée au temps du monde et irriguée par des flux (de capitaux, de modèles culturels, d'individus) globaux, ce qui tranche par ailleurs avec son urbanisation réelle caractérisée par le sous-équipement. A contrario, le quartier historique du Plateau dakarois, largement pourvu en équipements urbains, est aujourd'hui renvoyé à une idée de désuétude et de localisme, en particulier pour ce qui touche à sa vie nocturne très largement déclinante, voire moribonde. On perçoit ici ce que l'approche nocturne peut apporter à l'histoire urbaine d'une ville comme Dakar, sous l'angle notamment des dynamiques globales qui s'y projettent.

Outre l'entrée par les lieux, ou par les centralités et polarités urbaines, les manières de se vêtir, de parler et de danser, en somme, les styles culturels et les cultures matérielles qui sont mis en avant la nuit durant jouent également un rôle fondamental. À

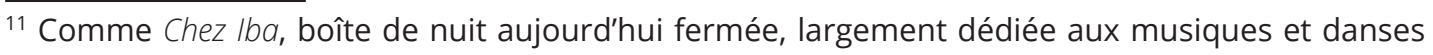
afro-cubaines, située dans le quartier de Dieuppeul. 
ce niveau, les infrastructures nocturnes de la ville deviennent en quelque sorte « incarnées» (people as infrastructure) (Simone, 2004), alors que les fictions de soi portées par les protagonistes de la nuit, et les jeunes femmes aventurières singulièrement, entrent en résonance avec l'environnement bâti, normé et organisé des établissements de nuit. Finalement, la somme complexe des lieux et de leurs usages, des interactions qui s'y trament et enfin des usages de soi, confère à la nuit urbaine valeur et fonction d'ailleurs social, que ce soit sur une base critique, stylistique ou plus sûrement à travers l'enchevêtrement des deux ${ }^{12}$. C'est notamment sous cette optique que l'on peut saisir comment l'indétermination propre à la nuit se voit attribuer une valeur fondamentalement positive, c'est-à-dire que l'incertain revêt un caractère productif.

\section{Productivité de l'incertain : nocturne liquide et mobilités}

- Je connais beaucoup de gens qui disent que Dakar mange les Hommes [du wolof «nit ñi » : humains]. C'est-à-dire que tu te fais bouffer complètement par les problèmes, que ce soit l'argent ou autre. Je veux dire, tous ces trucs que tu voudrais, mais dont tu sais très bien que ce n'est pas pour toi : être riche, respectée, célèbre, voyager... II y a aussi les gens qui font du n'importe quoi, qui pensent qu'ils peuvent t'imposer des trucs parce qu'ils sont plus forts que toi [physiquement et/ou socialement]. II y a de quoi devenir folle, en fait. [...] Ça te mange dans le sens que ça te bouffe la tête, tu n'as que des soucis. Partout où tu regardes, c'est la merde seulement, alors tu te dis qu'il n'y a rien pour toi ici. Tu te sens mal, tu n'es pas à l'aise, en fait.

- TF : Et quand tu sors la nuit?

- Voilà ! Pourquoi j'aime bien la nuit justement, c'est parce que c'est là que tu vis un peu quoi, c'est-à-dire, c'est à ce moment-là que tu as l'impression que ça vaut le coup de faire des choses. Tu peux toujours te faufiler dans les problèmes pour faire un peu comme tu as envie... Pour faire ce qui te plait, quoi. [...] Moi, je trouve que, dans un sens, la journée est dure [wolof : dëgër] alors que la nuit est molle [wolof : nooy] (rires). Tu vois, c'est pour dire que la nuit, on peut la manger, alors que la journée c'est elle qui va te bouffer à tous les coups : elle va te casser les dents, parce qu'elle est plus dure que toi. Par contre, la nuit, ce n'est pas la ville qui mange les Hommes, mais c'est plutôt les Hommes qui la croquent jusqu'à se faire exploser le ventre ${ }^{13}$ !

Les discours de ce type, très récurrents parmi mes interlocutrices « aventurières de la nuit », dévoilent ce que l'on pourrait nommer une identification temporelle, par analogie avec la question plus classique des identités spatiales (Lussault, 2003). La nuit est en effet présentée comme le temps où un engagement véritable - plaisant, satisfaisant, voire rassasiant - dans la vie de la cité est rendu possible. Elle en est pour ainsi dire la condition de possibilité temporelle et donc intrinsèquement temporaire. C'est en ce sens, notamment, que j’ai déjà eu à interpréter les nuits de la capitale sénégalaise en leur qualité « d'espace potentiel » (Fouquet, 2017). En se livrant ainsi aux potentialités

${ }^{12}$ Ce que j'ai nommé des « politiques du style » (Fouquet, 2015).

${ }^{13}$ Femme, 23 ans, Dakar, 2016. Entretien conduit en wolof et français. 
du nocturne - réelles, arguées ou désirées -, le problème est entre autres de trouver un moyen de composer avec les difficultés ou encore de résoudre les contradictions de façon créative, c'est-à-dire sans « complaisance soumise envers la réalité extérieure » selon Donald Winnicott (1975:127), à qui l'on doit le concept d'espace potentiel.

Par ailleurs, les propos de cette interlocutrice, mis en regard de ceux cités dans l'extrait qui précède, permettent de saisir l'enchevêtrement original de différents rapports au temps, ou de rapports à des temps différents : long, lent, distendu, voire suspendu, caractérisé par le présentisme et le waithood, d'une part ; bref, rapide, voire saccadé, lié à l'alternance diurne/nocturne et aux potentialités qui paraissent s'en dégager, d'autre part. Un paradoxe semble se dessiner à ce niveau : le fait d'embrasser l'indétermination du nocturne trouve une partie de sa raison d'être dans une tentative d'évitement de l'incertitude qui est celle, en l'espèce, que l'on peut relier à un horizon de vie perçu comme particulièrement nébuleux ou précaire. Il ressort de mes observations que certaines manières d'investir et de pratiquer les nuits urbaines permettent de résoudre ce type de « conflits temporels », ou tout au moins d'avoir prise sur eux et de les négocier. On comprend aussi que ce que l'on nomme génériquement l'incertain renvoie à des réalités ou des enjeux intrinsèquement hétérogènes et appelle une analyse plus fine, en regard des durées ou des temporalités qui lui donnent formes et sens d'une part, du point de vue des usages qui en sont fait d'autre part.

Suivant une ligne de pensée très proche, un aspect intéressant tient au jeu comparé du « dur » (diurne) et du « mou » (nocturne) formulé par l'interlocutrice citée plus haut, qui n'est pas sans évoquer l’idée de liquidité chère à Zygmunt Bauman (2007 ; 2013). Là encore, la fluidité associée aux positions occupées ou accessibles durant la nuit s'énonce par opposition avec la rigidité des systèmes de contraintes (économiques, sociales, morales) diurnes et l'âpreté des conditions de vie dans la cité. Sur la durée de mes enquêtes, j'ai pu observer que cette liquidité du nocturne se manifeste aussi à travers la relative confusion qu'elle induit quant aux rôles et statuts individuels. C'est là un aspect important pour comprendre certaines marges de manœuvre que les « aventurières » dakaroises parviennent à négocier à la faveur de la nuit. En raison même des transactions économico-sexuelles dans lesquelles elles sont engagées, ces jeunes femmes s'exposent frontalement au « stigmate de la putain » (Tabet, 1987 ; Pheterson, 2001). Il y a en ce sens nécessité d'entretenir un flou aussi important que possible sur leurs pratiques, en particulier celles se rapportant peu ou prou au travail du sexe. Mais le problème est également de garder le champ des possibles très largement ouvert, c'està-dire, notamment, se ménager les opportunités qui seraient à la fois les moins pénalisantes socialement et les plus profitables sur le plan matériel ou symbolique. En jouant ainsi constamment sur l'ambivalence des rôles et des positions sociales, en organisant une sorte d'anonymat statutaire, tout un éventail de places est dès lors accessible : de la prostituée à la petite amie, voire l'épouse en puissance. Il n'est pas rare qu'au cours d'une même nuit, des jeunes femmes endossent successivement ces rôles à la fois différents et complémentaires, en fonction à la fois des lieux (night-clubs branchés, restau- 
rants, bars-dancings spécialisés dans les rencontres de type prostitutionnel, etc.), des besoins et opportunités du moment, mais aussi, et peut-être surtout, des compétences relationnelles sur lesquelles chacune peut s'appuyer dans la gestion de ses nuits et des relations variées qu'elle y négocie. Ces compétences sont spécifiques à l'espace-temps nocturne en ce sens qu'elles font l'objet d'acquisitions au fil de " carrières de la nuit ", dès lors envisageables comme trajectoires d'apprentissages de savoirs pratiques (une intelligence des situations), mais aussi capitalisables plus durablement, notamment sur le plan socioculturel - assimilations linguistiques (français, anglais, italien, etc.) auprès de partenaires étrangers, savoir-faire et savoir-être dans une grande diversité de situations et de milieux sociaux, etc. Dans l'ensemble, l'incertitude est en quelque sorte aménagée puis utilisée comme une ressource, c'est-à-dire qu'elle est mise au service de stratégies individuelles de mobilité, mais aussi de « clandestinisation sociale (Fouquet, 2014c). De fait, les récits autobiographiques de mes interlocutrices font tous référence à un « avant » des carrières de la nuit, dominé par un sentiment d'impuissance et d'incapacité sociale et relevant en ce sens d'une sorte de soumission à la loi de l'incertain (et incidemment de l'indésiré), puis à un " après » où l'accumulation diachronique de savoirs (indexée aux expériences, rencontres, etc. qui fondent les « carrières de la nuit ») permettrait d'avoir davantage prise sur sa propre trajectoire et de la négocier au plus près de ses aspirations et intérêts. On rejoint ici l'idée voulant que l'incertain soit porteur d'une forme propre de productivité ou de créativité.

On pourrait mettre ces interprétations en regard de «liquéfactions » d'un autre type : celles qui caractérisent plus largement le secteur économique des loisirs nocturnes dakarois. En ce domaine, et à d'assez rares exceptions, plus rien ne semble encore pouvoir vieillir ou être soumis à la patine du temps. Les établissements de nuit ferment puis rouvrent à un rythme accéléré, avec de nouveaux propriétaires, sous un nouveau nom, avec une nouvelle décoration d'intérieur toujours plus dans la tendance, mais aussi rendue en quelque sorte froide ou aseptisée sous l'effet d'une certaine standardisation de l'offre. À noter que de tels phénomènes d'uniformisation de l'offre de loisirs urbains nocturnes n'ont rien de spécifique à Dakar ; ils ont été étudiés sous l'angle, entre autres, des effets du néolibéralisme sur les phénomènes de revalorisation urbaine et de gentrification (Chatterton, Hollands, 2003 ; Nofre, 2013), avec pour toile de fond les enjeux liés au déclin des centres urbains historiques. À ce jour, néanmoins, pas ou fort peu d'études ont été consacrées à ces questions en contextes urbains africains.

S'agissant ici du cas dakarois, on observe ainsi une succession de " nouveaux départs » ou de «nouveaux recommencements » (Bauman, 2013: 8-9), où tout ce qui n'est pas actuel - en tout cas considéré comme tel - est frappé de jugements de désuétude et ainsi voué à être mis au rebut pour éventuellement réapparaître sous le signe, encore et toujours, de la nouveauté. Ce critère du nouveau, ou du toujours plus actuel, constitue du reste un élément cardinal pour la définition subjective de la bonne ambiance parmi mes interlocuteurs. Il est même érigé en instrument marketing, comme l'explique ce 
professionnel des nuits dakaroises ayant assumé la direction de plusieurs boîtes de nuit renommées :

Le truc à savoir, c'est que les gens veulent toujours de la nouveauté, ils pensent que le nouveau c'est ce qu'il y a de meilleur. Dès qu'un nouvel endroit ouvre, tout le monde va se ruer là-bas. Ce qui fait que certaines boîtes ont un grand succès dès leur ouverture, mais déclinent rapidement. Dès qu'une autre boîte ouvre, les gens se déplacent encore vers le truc nouveau ${ }^{14}$.

Une interlocutrice « aventurière », que j’interrogeais sur ses préférences noctambules du moment, formule une réponse qui corrobore le goût ou la quête du nouveau qui se dessine ici :

C'est difficile de dire je préfère tel endroit, parce que ça change tout le temps... Ce qui est sûr en tout cas, c'est que pour qu'il y ait un peu de concurrence, il faut que les boîtes soient au top... Des trucs neufs, actuels quoi. Moi, c'est ça que je regarde avant d'aller à un endroit, si on a fait un effort pour le faire évoluer. C'est logique, t'as pas envie de te traîner dans des trucs que tu connais déjà par cœur ou qui sont trop anciens, trop sénégalais quoi ${ }^{15}$.

La recherche de la nouveauté s'accompagne d'usages sociaux répondant à un principe assez similaire : on " traverse » la nuit en l'arpentant de lieu en lieu ; on y butine des ambiances dans un déploiement parfois frénétique d'établissements de nuit visités (plutôt que fréquentés) et de circulations dans la ville conséquentes. Incidemment, la figure de l'« habitué.e » s'affaiblit alors que beaucoup n'ont plus pour habitude que d'être de passage, ce qui confère aux sociabilités une physionomie souvent diffuse ou atomisée : ce sont des sociabilités du mouvement autant que du moment, qui octroient dès lors une place centrale aux notions de rencontre (inopinée) et de croisement (furtif). La volatilité, voire l'inconsistance, qui caractérise ce secteur d'activités convoque ainsi une même idée de plasticité ou de liquidité propre à la nuit.

Deux éléments me paraissent finalement intéressants à souligner à ce niveau. D’abord, suivant les avis convergents d'une majorité de mes interlocuteurs « spécialistes pratiques » de la nuit à Dakar ${ }^{16}$, ces phénomènes de liquéfaction, tout au moins dans les formes évoquées ici, sont assez récents. Ils corroborent la transformation des centralités urbaines dakaroises déjà mentionnée avec, courant des années 1990, un déclin de la zone du Plateau - foyer historique des sociabilités urbaines y compris nocturnes - au bénéfice de la zone de Ngor-Almadies, où se concentrent désormais la majorité des bars et night-clubs. Toujours en accord avec ces avis informés, ce basculement dans la physionomie nocturne de la capitale sénégalaise serait consubstantiel à un virage néolibéral, relayé et même amplifié dans le champ politique national par l'arrivée au pouvoir d'Abdoulaye Wade en 2000, avec pour conséquences notamment la libéralisation de l'économie nocturne, l'ouverture massive de ce secteur - parmi

\footnotetext{
${ }^{14}$ Homme, 32 ans, Dakar, 2008. Entretien réalisé en français et wolof.

${ }^{15}$ Femme, 24 ans, Dakar, 2006. Discussion informelle en français et wolof.

${ }^{16}$ Notamment : élus municipaux au sein des zones de la ville concernées, entrepreneurs qui gèrent des établissements de nuit à Dakar depuis les années 1980, « professionnels de la nuit » type deejay ou barmen œuvrant également de longue date dans le secteur nocturne.
} 
d'autres - à des capitaux étrangers, mais aussi la mise en œuvre de grands travaux infrastructurels/routiers ayant permis le désenclavement de la zone Ngor-Almadies.

En écho à ces transformations, des « politiques de la nostalgie » (Lachenal, MbodjPouye, 2014) se manifestent. Nombreux sont ceux qui, à Dakar, déplorent ce qu'ils considèrent comme une perte d'authenticité des lieux et ambiances nocturnes, comme s'ils avaient cédé de leur épaisseur citadine à une logique marchande où les attentes et postures consuméristes prennent le pas sur tout le reste. Sont ainsi mis en regard et en opposition des lieux de sociabilité où l'on se rencontrait « vraiment » et où s'organisait quelque chose de l'ordre du moment partagé, d'une part, et des espaces que l'on perçoit comme uniquement dédiés au gisleen maa (du wolof : " m'as-tu vu »), c'est-à-dire où seul compte l'effet produit sur une assistance complice et faire-valoir d'un narcissisme compensateur, d'autre part. Ce sont entre autres les styles de plus en plus américanisés et bling-bling des jeunes publics des night-clubs en vue des Almadies qui sont ici dénoncés pour la superficialité dont ils seraient les marqueurs, non seulement à l'échelle restreinte de l'univers des loisirs nocturnes, mais en regard des évolutions de la société dakaroise, voire sénégalaise, bien plus généralement.

Les postures nostalgiques ici évoquées s'assimilent, de manière assez classique, à une critique sociale que l'on pourrait en premier lieu rattacher aux transformations urbaines rapides et profondes dont Dakar est le théâtre depuis une vingtaine d'années, alors que la physionomie de la ville, ses centres et points d'équilibre se remodèlent en permanence sous l'effet de travaux de voiries, de constructions de quartiers de standing où s'organise une séparation nette entre le privé et le public, de l'apparition de centres commerciaux et d'hypermarchés toujours plus nombreux - bref, d'un ensemble d'espaces impersonnels et non porteurs de passé dont on peine à faire « territoire » (Gervais-Lambony, 2003:140 et s.), au moins pour un temps donné. C’est non seulement le paysage et le « visage » de la ville qui s'en trouvent modifiés, mais aussi les manières que l'on a de s'y produire, d'y trouver et d'y projeter du sens citadin : « La forme d'une ville, Change plus vite, hélas ! que le cœur d'un mortel », écrivait Baudelaire (cité par Gervais-Lambony, 2012). De ce point de vue, la nostalgie s'affirme comme une expression possible de l'incertitude, en tant que « caractéristique des sociétés "inquiètes" de l'accélération des changements et de la perte de contrôle de leur destinée » (Ibid.).

La nostalgie comme mode intime de gestion de l'incertitude participe en ce sens à la production d'une géographie sensible, où les plaisirs (nocturnes) de la ville et leurs reconfigurations actuelles tiennent une place centrale. En filigrane de ces analyses, on voit également que toute une trame de la mobilité se retisse à celle de la nuit urbaine ; a minima, l'urbain nocturne s'énonce dans les termes de la mobilité et, réciproquement, la mobilité est nettement reliée à l'expérience nocturne. Dans un texte considéré comme pionnier pour l'étude de la nuit, Murray Melbin a proposé d'« appréhender la nuit comme une nouvelle frontière (frontier), au sens où l'expansion dans les heures sombres serait la continuation des migrations [vers l'Ouest américain] » (1978 : 3) ; il est 
donc intéressant de voir que, dès ce texte fondateur, nocturnité et mobilité sont mises en regard l'une de l'autre.

Sur mon terrain dakarois, cette approche croisée se justifie plus spécifiquement à au moins deux égards. D’une part, les enjeux de mobilité - sociale comme géographique prennent une force particulière parmi de jeunes Sénégalais massivement confrontés à des situations où les sentiments de stagnation sociale et de blocage géographique prévalent. Or, je l'ai dit, certains usages des nuits dakaroises se rapportent directement à ces expériences de l'immobilisme forcé. D'autre part, et j'insisterai davantage sur ce point, mobilité et nocturnité se rejoignent sur un plan plus conceptuel ou intellectuel, en particulier au chapitre de l'indétermination. À cet égard, je suggère que la nuit urbaine constitue un espace-temps à forte valeur de mobilité ajoutée pour ce qui touche, en particulier, à l'indétermination propre aux situations de changements en train de se faire.

- Qu'est-ce qui me plaît la nuit? C'est que, quand tu sors, tu ne sais pas très bien ce qui va arriver. Je veux dire : tu sais plus ou moins où tu vas, avec qui, pour faire quoi... Mais bon, même si tu as un peu prévu tout ça, il y a toujours quelque chose qui va arriver, quelqu'un que tu vas rencontrer, ou je ne sais pas quoi encore, qui va transformer ce que tu avais prévu. Tu te dis au départ « je vais de $A$ à $B$ ou à $C$, et basta ", et en fait tu vas te retrouver à $X, Y$ ou $Z$, et tu ne sais même pas comment c'est possible. Je veux dire, il t'est arrivé tellement de trucs pour arriver à cet endroit-là à la fin, que tu n'arrives même plus à t'en rappeler. - TF : La nuit, c'est «tukki réer ${ }^{17}$ » alors?

- Oui oui, tukki réer. Ou plutôt zigzag rekk [zigzaguer seulement] ! Tu as perdu ta route, oui, mais c'est plutôt que, dans un sens, tu prends un chemin [nocturne] qui n'est pas très bien tracé, quoi. Tu fabriques un peu ta voie pendant même que tu marches. Je ne sais pas... C'est ça pour moi, la nuit, c'est tout le temps du woorul woorul [de l'incertain], quoi ! Des fois, ça va être super bien, des fois c'est pas bien... des fois c'est vraiment pas bien (rires). Mais en tous cas, ce qui est sûr : c'est jamais exactement comme tu avais pensé ${ }^{\prime 8}$.

On comprend à travers ces mots qu'il y a une espèce d'heuristique de l'incertitude dont il faut bien prendre la mesure, et qui relève par ailleurs intrinsèquement du registre de la mobilité - réelle, imaginée ou encore désirée. Tout ceci renvoie au régime de l'incertain et de l'indéterminé que la nuit, comme la mobilité, emporte avec elle. On peut en référer tout d'abord à la nature même de l'expérience sensible et sensorielle de la nuit, caractérisée par un bouleversement des repères visuels, olfactifs, auditifs, du toucher, etc. Les transformations cognitives ainsi induites pèsent sur les manières individuelles d'interpréter les situations et de s'y impliquer en conséquence. Indétermination sociale, morale et politique également, alors que les règles diurnes dominantes sont d'une certaine façon altérées ou tout au moins reconfigurées par la

\footnotetext{
17 Cette formule wolof de tukki réer (littéralement : se perdre en voyageant) renvoie à l'idée d'une perte de repères sociaux, culturels, voire identitaires, qui est généralement associée à l'expérience migratoire dans les discours et imaginaires sociaux au Sénégal.

18 Femme, 24 ans, Dakar, 2017. Entretien traduit du wolof.
} 
nuit. Enfin, indétermination subjectivement construite et recherchée qui, parmi mes interlocutrices à Dakar, renvoie à la possibilité d'instaurer l'incertain en stratégie, mais aussi d'initier d'autres trajectoires en dehors des chemins les plus normés. C'est ainsi que s'organise une forme bien spécifique de productivité de l'incertain en contextes nocturnes, qui sous-tend des usages originaux de l'espace-temps urbain.

\section{Conclusion : l'incertain et l'indéterminé comme leviers critiques}

Les configurations discutées dans cette étude à la fois révèlent et relèvent d'un rapport spécifique au réel, ou plus exactement d'une articulation bien particulière des catégories du possible et du réel. Henri Bergson (1938), dans une réflexion qu'il a dédiée à ce thème, a suggéré que le possible est plus que le réel, car il y surajoute un acte de l'esprit. Le réel renvoie à la finitude de ce qui advient effectivement - ou de ce qui est effectivement advenu ; le possible, quant à lui, resitue ce réel dans un horizon autrement plus vaste. Il est intellectuellement, mais aussi peut-être socialement, voire politiquement, beaucoup plus riche et dense. Cet acte de l'esprit, ce petit ou ce grand truc en plus, on pourrait bien sûr le situer dans le registre de l'imaginaire. C'est-à-dire l'imagination d'autres possibles, alternatifs à ceux qui s'actualisent dans la durée et la répétition de l'expérience.

Or, nous en revenons ici à ce qui peut être considéré comme une autre caractéristique de la nuit : le fait non seulement qu'elle polarise largement les imaginaires sociaux (sur le mode négatif de la menace, ou positif d'un autre monde à venir ou à saisir), mais aussi qu'elle participe d'un renforcement du rôle de l'imagination. Elle est en quelque sorte le réel plus un « travail de l'imagination » (Appadurai, 2001), qui vient se suppléer aux évidences, aux présupposés voire aux certitudes que le diurne porte en son sein. C'est cette idée que le rêve est au bout de la nuit... chaque soir. Idée que je retrouve fréquemment, pour ne pas dire en permanence, parmi mes interlocutrices à Dakar et qui fonctionne comme un véritable moteur pour repartir à l'aventure, nuit après nuit, quoi qu'il en soit des fortunes, bonnes ou mauvaises, de la nuit précédente. De ce point de vue, les expériences nocturnes envisagées dans cette étude sont placées sous le signe du potentiel, du productif et du mobile, a contrario de la journée qui est vécue sur le mode de l'immobilisme, du banal et donc du prévisible dans ce qu'il a de plus fâcheux avec l'idée cette fois-ci que, jour après jour, seul le pire est au rendez-vous. Mobilité et nocturnité, là encore, se rejoignent et se renforcent mutuellement à travers cette idée du déplacement (social et/ou géographique) comme potentialité. Et c'est peut-être même cette virtualité, ou cette dimension imaginaire surajoutée, qui caractérise le mieux la mobilité par rapport au seul déplacement physique - mais aussi le nocturne, par contraste avec la souvent rude et stricte tangibilité du diurne.

C'est également à ce niveau que s'écrit la partition critique de la nuit. Embrasser l'incertain, se livrer à la rencontre de l'inconnu et de l'imprévu, c'est favoriser le potentiel - les risques, mais aussi les possibles qu'il contient - plutôt que la sécurité du prévisible. En ce sens, le fait de réinvestir l’incertain et l’indéterminé de cette valeur positive com- 
porte et implique une forme de résistance. On pourrait considérer que cela fait entrer cette dimension d'incertitude du nocturne dans le registre du politique, à petits pas en quelque sorte. Le potentiel critique de la nuit tiendrait ainsi à la résistance qu'elle oppose de facto aux injonctions de clarté et de sûreté du jour : le possible nocturne ferait dès lors défi au réel diurne.

\section{Bibliographie}

Appadurai A. (2001), Après le colonialisme. Les conséquences culturelles de la globalisation, Paris, Payot.

Appadurai A. (2004), « The Capacity to Aspire: Culture and the Terms of Recognition », in V. Rao, M. Walton (dir.), Culture and Public Action, Palo Alto, Stanford University Press, p. 59-84.

Bauman Z. (2007), Liquid Times. Living in an Age of Uncertainty, Londres, Polity Press.

Bauman Z. (2013), La vie liquide, Paris, Fayard (« Pluriel»).

Bergson H. (1938), La pensée et le mouvant, Paris, Presses universitaires de France.

Bredeloup S. (2014), Migrations d'aventures. Terrains africains, Paris, Éditions du Comité des travaux historiques et scientifiques.

Cabantous A. (2009), Histoire de la nuit, XVII $-X V I I I^{e}$ siècles, Paris, Fayard.

Chaouad R. (2016), «Émergence : genèse et circulation d'une notion devenue catégorie d'analyse », Revue internationale et stratégique, $n^{\circ} 103$, p. 55-66.

Chatterton R. (2002), « Governing Nightlife: Profit, Fun and (Dis)Order in the Contemporary City », Entertainment Law, vol. 1, n² 2, p. 23-49.

Chatterton R., Hollands P. (2003) (dir.), Urban Nightscapes. Youth Cultures, Pleasure Spaces and Corporate Power, London, Routledge.

CoHen S. (2002 [1972]), Folk Devils and Moral Panics: The Creation of the Mods and Rockers, London, Routledge.

Cooper E., Pratten D. (2015), Ethnographies of Uncertainty in Africa, London, Palgrave Macmillan.

Cruise O’Brien D., Diop M. C., Diouf M. (2002), La construction de l'État au Sénégal, Paris, Karthala.

Diouf M. (2013), « Les jeunes dakarois, la scène urbaine et le temps du monde à la fin du XX siècle », in M. Diouf, R. Fredericks (dir.), Les arts de la citoyenneté au Sénégal, Paris, Karthala, p. 49-91.

Fouquet T. (2008), « Migrations et "glocalisation” dakaroises », in M. C. Diop (dir.), Le Sénégal des migrations. Mobilités, identités et sociétés, Paris, Karthala/Crepos, p. 241273.

Fouquet T. (2011), Filles de la nuit, aventurières de la cité. Arts de la citadinité et désirs de l'Ailleurs à Dakar, thèse de doctorat en anthropologie, Paris, EHESS. 
FoUQUET T. (2013), «Esquisses d'un art de la citadinité subalterne : les aventurières de la nuit dakaroise », in M. Diouf, R. Fredericks (dir.), Les arts de la citoyenneté au Sénégal, Paris, Karthala, p. 131-157.

Fouquet T. (2014a), «Penser au-delà du terrain sexuel à Dakar : éléments d'une épistémologie située », Questions de Recherche, n 44, Paris, Sciences Po-CERI, 20 p.

Fouquet T. (2014b), « Introduction au thème : Construire la Blackness depuis l'Afrique, un renversement heuristique ", Politique africaine, $\mathrm{n}^{\circ} 136$ (dossier thématique « Blackness. Circulation des mondes atlantiques et conscience noire en Afrique »), p. 5-19.

FouquET T. (2014c), « La clandestinité comme stratégie. Sur la sexualité transactionnelle à Dakar », in C. Broqua, C. Deschamps (dir.), L'échange économicosexuel, Paris, Éditions de l'EHESS, p. 125-152.

FOUQUET T. (2015), « La trame politique des cultures urbaines : motifs dakarois », in M. Cahen, M.-E. Pommerolle, E. K. Tall (dir.), Collective Mobilisations in Africa/ Mobilisations collectives en Afrique, Leiden/Boston, Brill, p. 112-141.

Fouquet T. (2016), « Politiques de la nuit et paysages nocturnes de la ville. Perspectives ouest-africaines », Sociétés politiques comparées, $n^{\circ} 38$ (dossier thématique « Nuits urbaines »), p. 1-16.

FOUQUET T. (2017), « La nuit urbaine, un espace potentiel ? Hypothèses dakaroises », Cultures e conflits, $n^{\circ} 105-106$, p. 83-97.

Fouquet T. (2018), « Grassroots Cosmopolitics: Critical Notes on a Cosmopolitan Africa », African Identities, vol. $16, \mathrm{n}^{\circ}$ 2, p. 219-230.

FouRCHARD L. (2004), «L'histoire urbaine en Afrique : une perspective ouest-africaine », Histoire urbaine, $\mathrm{n}^{\circ}$ 9, p. 129-144.

GaOnKar D. P. (dir.) (2001), Alternative Modernities, Durham/London, Duke University Press.

Geschiere P., Rowlands M. (1996), « The domestication of Modernity: Different Trajectories », Africa, vol. 66, $\mathrm{n}^{\circ}$ 4, p. 552-554.

Gervais-Lambony P. (2003), Territoires citadins : 4 villes africaines, Paris, Belin.

Gervais-Lambony P. (2012), " Nostalgies citadines en Afrique Sud », EspacesTemps.net. En ligne, consulté le 14 novembre 2019. URL : http://www.espacestemps.net/ document9459.html.

Hannerz U. (1990), « Cosmopolitans and Locals in World culture », Theory, culture and society, vol. 7, $\mathrm{n}^{\circ}$ 2, p. 237-251.

Honwana H. (2012), The Time of Youth: Work, Social Change and Politics, Herndon, VA, Kumarian Press.

Hartog F. (2003), Régimes d’historicité. Présentisme et expérience du temps, Paris, Le Seuil. IRwin J. (1977), Scenes, Beverly Hills, Sage. 
Kleist N., Thorsen D. (2017), Hope and Uncertainty in Contemporary African Migration, New York/London, Routledge Studies in Anthropology.

LABussière O. (2013), "Flux, ambiances et ré-enchantement du monde », Ambiances. En ligne, consulté le 14 novembre 2019. URL : http://journals.openedition.org/ ambiances/141.

Lachenal G., Mbodj-Pouye A. (dir.) (2014), « Introduction au thème : Restes du développement et traces de la modernité en Afrique », Politique africaine, $\mathrm{n}^{\circ} 135$ (dossier thématique « Politiques de la nostalgie »), p. 5-21.

Lussault M. (2003), «Identité spatiale», in J. Levy, M. Lussault, Dictionnaire de la géographie et de l'espace des sociétés, Paris, Belin.

Melbin M. (1978), « Night as Frontier », American Sociological Review, vol. 43, p. 3-22.

Mrembe A. (2006), « La colonie : son petit secret et sa part maudite», Politique africaine, $\mathrm{n}^{\circ} 102$, p. 101-127.

Mitchell C. (1996 [1956]), « The Kalela Dance/La danse du kalela. Aspects des relations sociales chez les citadins africains en Rhodésie du Nord », Enquête, n 4, p. 213-243.

Nativel D. (2016), « Les sens de la nuit. Enquête sur des sensorialités urbaines coloniales à Madagascar et au Mozambique ", Sociétés politiques comparées, nº 38 (dossier thématique « Nuits urbaines »), p. 1-15.

Nofre J. (2013), «Vintage Nightlife: Gentrifying Lisbon downtown», Fennia: International Journal of Geography, vol. 2, n 191, p. 106-121.

PAQUOT T. (2000), « Le sentiment de la nuit urbaine aux XIX et XX ${ }^{\mathrm{e}}$ siècles », Les annales de la recherche urbaine, $\mathrm{n}^{\circ}$ 87, 2000, p. 6-14.

Pena-Vega A. (2014), «À l'épreuve des incertitudes », Communications, vol. 95, nº 2, p. 5-8.

Pheterson G. (2001), Le prisme de la prostitution, Paris, L'Harmattan.

Sardar Z. (2010), «Welcome to Postnormal Times », Futures, nº 42, p. 435-444.

Simone A. (2004), « People as Infrastructure: Intersecting Fragments in Johannesburg », Public Culture, vol. 16, n 3, p. 407-429.

Simone A. (2005-2006), « Atteindre des mondes plus vastes », Politique africaine, $\mathrm{n}^{\circ} 100$, p. 36-53.

TABET P. (1987), « Du don au tarif. Les relations sexuelles impliquant compensation », Les Temps modernes, $\mathrm{n}^{\circ}$ 490, p. 1-53.

Vidal L., Musset A. (dir.) (2015), Les territoires de l'attente. Migrations et mobilités dans les Amériques (XIXe - XXIe siècle), Rennes, Presses universitaires de Rennes.

Weber M. (2014), La ville, Paris, La Découverte (« Politique et sociétés »).

Winnicott D. W. (1975), Jeu et réalité. L'espace potentiel, Paris, Gallimard. 